\title{
Clinical Impact of 99mTc-MAA SPECT/CT-based Personalized Predictive Dosimetry in Selective Internal Radiotherapy: a Retrospective, Single-center Study for Unresectable HCC Patients
}

\section{Ana-Maria Bucalau ( $\square$ Ana-Maria.Bucalau@erasme.ulb.ac.be )}

Centre Universitair Bruxelles Hôpital Erasme: Hopital Erasme https://orcid.org/0000-0003-0444-7806

\section{Benoît Collette}

Centre Universitair Bruxelles Hôpital Erasme: Hopital Erasme

Illario Tancredi

Centre Universitair Bruxelles Hôpital Erasme: Hopital Erasme

Michael Vouche

Jules Bordet Institute: Institut Jules Bordet

Martina Pezzullo

Centre Universitair Bruxelles Hôpital Erasme: Hopital Erasme

Jason Bouziotis

Centre Universitair Bruxelles Hôpital Erasme: Hopital Erasme

Rodrigo Moreno-Reyes

Centre Universitair Bruxelles Hôpital Erasme: Hopital Erasme

Nicola Trotta

Centre Universitair Bruxelles Hôpital Erasme: Hopital Erasme

Jean Luc Van Laethem

Centre Universitair Bruxelles Hôpital Erasme: Hopital Erasme

Gontran Verset

Centre Universitair Bruxelles Hôpital Erasme: Hopital Erasme

\section{Research Article}

Keywords: Selective Internal RadioTherapy, Transarterial radioembolization, Hepatocellular carcinoma, 99mTc-MAA scintigraphy

Posted Date: November 30th, 2021

DOI: https://doi.org/10.21203/rs.3.rs-1111102/v1 
License: (c) (i) This work is licensed under a Creative Commons Attribution 4.0 International License. Read Full License 


\section{Abstract}

\section{Background}

Recent data indicates that personalized dosimetry-based selective internal radiotherapy (SIRT) may be associated with better outcome for unresectable hepatocellular carcinoma (HCC).

\section{Aim}

We aim to evaluate the contribution of personalized predictive dosimetry (performed with Simplicity $90 \AA$ software) in HCC patients by comparing them to our historical cohort whose activity was determined by standard dosimetry.

\section{Methods}

This is a retrospective, single-center study conducted between February 2016 and December 2020 that included patients with HCC who received SIRT after simulation based on either standard dosimetry (group A) or, as of December 2017, on personalized dosimetry (group B). Primary objectives were best overall response (BOR) and objective response rate (ORR) evaluated by mRECIST at 3 months. Safety and toxicity profiles were evaluated at day 1, 1- and 3-months post-treatment. For group A we studied the dose-response relationship at 3 months and compared the activity to be administered determined a posteriori using Simplicit $90{ }^{\circledR}$ and the activity actually administered determined by the standard approach.

\section{Results}

Between February 2016 and December 2020, 66 patients received 69 simulations leading to 40 treatments. The median follow-up time was equal for both groups, 21 months (range 3-55) in group A and 21 months (range 4-39) in group B. The per patient analysis revealed a significant benefit of personalized predictive dosimetry in terms of better overall response at 3 months ( $80 \%$ vs. $33.3 \%, p=0.007)$ and at 6 months $(77.8 \%$ vs. $22.2 \%, p=0.06)$. This trend was found in the analysis by nodule with a response rate according to mRECIST of $87.5 \%$ for personalized dosimetry versus $68.4 \%$ for standard dosimetry at 3 months, $p=0.24$. Only one grade 3 biological toxicity (hyperbilirubinemia) was noted in group $A$. The comparison between the administered activity and the recommended activity recalculated a posteriori using Simplicit90Y $\circledast$ showed that the vast majority of patients who progressed (83.33\%) received less activity than that recommended by the personalized approach or an inadequate distribution of the administered activity.

\section{Conclusions}

Our study confirms that the use of personalized dosimetry allows a better selection of HCC patients who can benefit from SIRT, and consequently, improves the effectiveness of this treatment. 


\section{Introduction}

Hepatocellular carcinoma (HCC) accounts for $90 \%$ of all primary liver cancers, with the majority of cases being associated to cirrhosis ${ }^{1}$. Therefore choosing the most suitable treatment option depends not only on the tumor stage, but also on the severity of the underlying liver disease. Current guidelines ${ }^{1,2}$ consider the Barcelona Clinic Liver Cancer (BCLC) staging system as the algorithm of choice for tumor staging and therapeutic options ${ }^{3}$, taking into account tumor burden, Child-Pugh classification and performance status. Locally advanced HCC is suitable for transarterial locoregional therapies, conducted mostly in a palliative setting.

Selective Internal RadioTherapy (SIRT), known as TransArterial RadioEmbolization (TARE) in the context of liver disease, consists here of the intra-arterial infusion of small beads that are loaded with a radioactive isotope, generally Yttrium-90 $\left({ }^{90} \mathrm{Y}\right)$ or more recently Holmium-166 $\left({ }^{166} \mathrm{Ho}\right)$, and it relies on the beta radiation emitted by the isotope to induce tumor necrosis, with a minor contribution from microembolization ${ }^{4}$. Its efficacy was reported in several large patient series ${ }^{5-8}$. Two products are commercially available for ${ }^{90} \mathrm{Y}$-based SIRT. ${ }^{90} \mathrm{Y}$-labelled resin microspheres (SIR-Spheres; Sirtex Medical, Sydney) and ${ }^{90} \mathrm{Y}$ glass microspheres (TheraSphere ${ }^{\circledR}$; Boston Scientific, Marlborough, MA, USA) are approved for the treatment of unresectable liver tumors, including HCC. Currently, new ${ }^{166} \mathrm{Ho}$-PLLA microspheres (QuiremSpheres ${ }^{\circledR}$ ), aiming to improve work-up (or simulation) by the use of ${ }^{166} \mathrm{Ho}$-PLLA "scout" activity, have been developed and showed safety and efficacy in unresectable, chemorefractory liver metastases 9,10 .

Nevertheless, for advanced tumors or chemoembolization refractory HCC, two RCT phase III, randomized controlled trials, comparing SIRT to Sorafenib, showed no difference in terms of overall survival (OS) ${ }^{11,12}$ Moreover, the addition of SIRT to Sorafenib, evaluated in a recent phase II study, did not show benefit in terms of survival ${ }^{13}$.

Usually, the prescribed activity in these trials was based on the body surface area (BSA). This represents a kind of standard calculation method for the activity of ${ }^{90} \mathrm{Y}$ to be administered and is assumed to correlate with the patient's liver volume, adjusted by the percentage of tumor involvement and the magnitude of the lung-shunt fraction (i.e. the fraction of injected microspheres lodged within the precapillary of the lungs) ${ }^{14,15}$.

Nevertheless, the BSA method may lead to underdosing (e.g., large livers) due to its moderate correlation to the liver volume and the percentage of tumor involvement adds little value when adjusting the activity to administer.

In order to optimize the administered activity, the concept of a more personalized dosimetry has been advocated, making use of patient-specific parameters and a multi-compartmental modeling that estimates the dose to the tumor, based on the Medical Internal Radiation Dose (MIRD) model. 
The capacity to predict the distribution of ${ }^{90} \mathrm{Y}$-microspheres has been shown to be a factor of improvement of SIRT efficacy ${ }^{16-18}$. The similar distribution of ${ }^{99 \mathrm{~m}} \mathrm{Tc}$-macroaggregated albumin $\left({ }^{99 \mathrm{~m}} \mathrm{Tc}\right.$ MAA) and ${ }^{90} \mathrm{Y}$-microspheres allows dosimetry simulation using ${ }^{99 \mathrm{~m}}$ Tc-MAA single-photon emission computed tomography ( ${ }^{99 \mathrm{~m}} \mathrm{Tc}-\mathrm{MAA}$ SPECT /CT) co-registered with MRI, or contrast-enhanced CT (CE-CT).

In our institution glass microspheres (TheraSphere ${ }^{\circledR}$, Boston Scientific) have been used since 2016 at first using a standard dosimetry-based simulation, and, more recently, with a personalized dosimetry to prescribe a more accurate activity (i.e.: calculated using Simplicit ${ }^{90} \mathrm{Y}^{\mathrm{TM}}$, a software able to do multicompartmental MIRD dosimetry). According to recent publications, a (mean) absorbed dose of, at least, 205 Gy delivered to the lesion is required to achieve an optimal response without exceeding 70 to $75 \mathrm{~Gy}$ to the non-tumoral liver ${ }^{19,20}$.

Hereby, we report our data regarding the use and potential benefits of personalized dosimetry for SIRT in our tertiary Hospital, by retrospectively comparing the response rate of SIRT obtained using standard dosimetry-based simulation to this obtained with SIRT using personalized dosimetry-based simulation. In addition, in patients treated after standard-dosimetry-based simulation, we compared the activity administered and the activity that would have been administered if personalized dosimetry had been applied. Finally, we analyzed the safety of SIRT in these two groups as well as the impact of personalized dosimetry had on survival.

\section{Material And Methods}

\section{a. Study design and patient allocation}

This is a retrospective study conducted in a tertiary health-center in Belgium, from February 2016 to December 2020, that enrolled 66 consecutive HCC patients who underwent at least one work-up (based on ${ }^{99 m}$ Tc-MAA scintigraphy) for radioembolization and received or not the treatment by SIRT (by Y90loaded glass microspheres). Patients were divided in two consecutive groups. Twenty-nine patients underwent work-up with standard dosimetry-based simulation (group A) from February 2016 to November 2017 and 37 patients underwent work-up with personalized dosimetry-based simulation (group B) from December 2017 to last enrolment at the end of 2020 (Figure 1).

The study was reviewed and approved by the local ethics committee (P2021/221). Due to the retrospective nature of the study, informed consent was not required.

\section{b. Patients' selection and population}

Study participants presented naive or previously treated HCC diagnosed by noninvasive criteria for cirrhotic patients, using contrast-enhanced MRI (CE-MRI) or contrast-enhanced computed tomography (CECT) or by pathology confirmation for noncirrhotic patients, according to European Association for the Study of the Liver/American Association for the Study of Liver Diseases guidelines. 
Patients were referred from the multidisciplinary hepatology tumor board and met the following criteria for inclusion: unresectable HCC not eligible for curative treatments (ablative treatments or surgical resection) with at least one measurable lesion, liver dominant or liver only disease; Child-Pugh score $\leq$ B7, Eastern Cooperative Oncology Group (ECOG) Performance Status score $\leq 1$. Exclusion criteria included: lung absorbed dose > 30 Gy or uncorrectable extrahepatic deposition of the MAA activity identified at ${ }^{99 m}$ Tc-MAA (whole body) scintigraphy, unmanageable intolerance to contrast medium and contraindication to hepatic angiography.

\section{c. Study outcomes}

The co-primary objectives of the study were the comparison of the objective response rate (ORR evaluated by modified Response Evaluation Criteria in Solid Tumors [MRECIST] ${ }^{21}$, defined by the proportion of treated nodules that presented complete or partial response, as well as the best overall response (BOR) defined as the best recorded response per patient from the start of the study treatment until disease progression between the two groups of patients (i.e., group A treated by ${ }^{90} \mathrm{Y}$ with an activity calculated using standard predictive dosimetry and group B using personalized predictive dosimetry).

The secondary objectives were: (1) the comparison of the two treatment groups in terms of progression free survival (PFS), defined as the time from treatment to the first observation of progressive disease or death, and overall survival (OS), defined as the time from treatment to death of any cause; (2) safety and toxicity profiles in the two groups evaluated according to the Common terminology Criteria of Adverse Events Version 5.0 (CTCAE V5.0) ${ }^{22}$; safety was evaluated clinically and biologically at 24 hours, 1 and 3 months and radiological adverse events (AEs) were recorded at 3 months after treatment; (3) for group A: (i) dose-response link investigation using mRECIST criteria on target lesions at 3 months, (ii) comparison between the activity to be administered by SIRT determined using a personalized dosimetry software with multicompartment MIRD technique (Simplicit ${ }^{90}{ }^{\circledR}$ ) to the activity administered determined by the classical non-compartmental dosimetry planning, using a target volume based on the ${ }^{99 \mathrm{~m}} \mathrm{Tc}-\mathrm{MAA}$ SPECT only (for patients treated before the acquisition of Simplicit ${ }^{90} \mathrm{Y}{ }^{\circledR}$ ); (4) for group B, dose-response link investigation using mRECIST (target) criteria at 3 months.

\section{d. Study procedures, activity calculation and dosimetry}

The radioembolization procedure was performed over two different sessions: work-up session and treatment session, by the same two interventional radiologists with 5-10 years' experience (between 50100 procedures per year). This work-up evaluation started with an angiography in order to obtain a precise map of the patients' abdominal vascular anatomy and coil embolization was performed if gastrointestinal branches arising from the hepatic arteries were found.

Patients in group A received treatment with ${ }^{90} \mathrm{Y}$ activity calculation based on the classical noncompartmental MIRD dosimetry planning following standard guidelines, using a volume based on the ${ }^{99 m}$ TC-MAA SPECT/CT. 148 to $185 \mathrm{MBq}$ of ${ }^{99 \mathrm{~m}} \mathrm{TC}-\mathrm{MAA}$ were injected after diagnostic angiography in the hepatic artery. Images were performed on a Philips BrightView XCT gamma-camera with a Low Energy 
High Resolution (LEHR) collimator. First, a whole-body scan was acquired to determine lung shunting $(140 \pm 14 \mathrm{keV}, 18 \mathrm{~cm} / \mathrm{min}, 256$ pixels wide). Then, a liver-centered SPECT/CT was conducted to estimate the spatial distribution of the ${ }^{99 \mathrm{~m}} \mathrm{Tc}-\mathrm{MAA}\left(140 \pm 14 \mathrm{keV}, 32\right.$ projections, $30 \mathrm{~s} /$ projection, $360^{\circ}, 128 \times 128$ pixels). The iterative reconstruction method commercially available used is Astonish $₫$ (3 iterations, 8 subsets). A calculation sheet from the ${ }^{90} \mathrm{Y}$ provider gave us the activity knowing the volume segmented from the ${ }^{99 m}$ Tc-MAA SPECT data (with thresholding based on a maximum intensity percentage), the lung shunt fraction determined on the whole-body scan (with manual segmentation), and the desired dose to the targeted volume (based on standard guidelines). ${ }^{99 m}$ Tc-MAA lung shunt fraction did not exceed 30 Gy in a single treatment or $50 \mathrm{~Gy}$ in case of multiple treatments. In case on an unfavorable ${ }^{99 m}$ Tc-MAA workup, the procedure was repeated and a solution was searched for (e.g., more selective placement of the catheter during injection to improve the targeting of the lesion). If ${ }^{90} \mathrm{Y}$-based SIRT could not be performed, the patient was treated according to best medical practice. If the work-up had a favorable outcome, the patients were re-admitted for treatment within 15 days.

${ }^{90} \mathrm{Y}$ Bremsstrahlung Emission Computed Tomography ( ${ }^{90} \mathrm{Y}$ BECT/CT) post-treatment images used for dosimetry purposes were acquired on the same Philips BrightView XCT gamma-camera. 0,42 to 5,1 GBq were injected regarding this standard predictive dosimetry group (A). An energy window around $120 \mathrm{keV} \pm$ $24 \mathrm{keV}$ was chosen to avoid the lead fluorescence $\mathrm{X}$-rays around $80 \mathrm{keV}$ and more energetic photons, eventually passing through the collimator because Medium Energy General Purpose (MEGP) collimator was used. First, a whole-body scan was acquired to visually confirm the absence of lung shunting (120 $\mathrm{kev} \pm 24 \mathrm{keV}, 12 \mathrm{~cm} / \mathrm{min}, 256$ pixels wide). Then, a liver-centred BECT/CT was conducted to estimate the spatial distribution of ${ }^{90} \mathrm{Y}$-TheraSphere ${ }^{\circledR}\left(120 \mathrm{kev} \pm 24 \mathrm{keV}, 64\right.$ projections, $30 \mathrm{~s} /$ projection, $360^{\circ}, 64 \times 64$ pixels). The same commercially available algorithm as previously described was used to reconstruct the data.

In order to compare the received activity according to standard predictive dosimetry and the activity that would have been recommended by the Simplicit ${ }^{90}{ }^{\circledR}$ software, patients in group $A$ for which we disposed of imaging at 3 months after treatment, were included. For all of them, CE-MRI, or CECT, ${ }^{99 m}$ Tc-MAA SPECT/CT and ${ }^{90} \mathrm{Y}$ BECT/CT were available. Radiological tumor response, evaluated following the mRECIST target criteria at 3 months, was correlated with the perfused tumor dose and the perfused fraction of the total tumor volume, determined using ${ }^{90} \mathrm{Y}$ BECT/CT and Simplicit ${ }^{90} \mathrm{Y} \circledast$. We also calculated the activity needed to reach 205 Gy in the perfused tumor, using ${ }^{99 m}$ Tc-MAA SPECT/CT and Simplicit ${ }^{90} Y \circledR$. The activity to be administered based on this minimal tumor absorbed dose criteria was compared to the activity actually administered. We plotted the relative differences, and correlated these differences with the radiological tumor response.

Patients in group $B$ received treatment after personalized predictive dosimetry that was performed using Simplicit ${ }^{90}{ }^{\circledR}$ software. MRI or CECT were used for the segmentation of the liver, the tumor, and the nontumoral liver (manually on MRI, automatically on CE-CT with corrections when needed). Then, ${ }^{99 m}$ Tc-MAA SPECT/CT was co-registered (rigid or non-rigid coregistration when needed) and the perfused volume 
determined (with thresholding based on a maximum intensity percentage), after lung shunt fraction evaluation on the whole-body scan. Acquisition parameters are the same as described above.

Regarding post-treatments imaging, in group $B,{ }^{90} \mathrm{Y}$ PET/CT were available for 9 patients. ${ }^{90} \mathrm{Y}$ PET/CT post-treatment images used here for dosimetry purposes were acquired on a digital Philips Vereos PET/CT scanner (20 minutes per bed position for a total of 40 minutes or 2 bed positions, $288 \times 288$ pixels of $2 \times 2 \mathrm{~mm}$ with a slice thickness of $2 \mathrm{~mm}$ ). The iterative reconstruction algorithm is an Ordered Subset Expectation Maximization (OSEM, 3 iterations, 17 subsets, with Point Spread Function option applied). Personalized dosimetry was performed using Simplicit ${ }^{90} Y{ }^{\circledR}$. MRI or CECT were used to do the segmentation of the liver, the tumor, and the non-tumoral liver (manually on MRI, automatically on CECT with corrections when needed). Then, ${ }^{90} \mathrm{Y} \mathrm{PET} / \mathrm{CT}$ was co-registered (rigid or non-rigid coregistration when needed) and the perfused volume determined (with thresholding based on a maximum intensity percentage). Lung shunt fraction was evaluated on a Bremsstrahlung Emission Whole Body scan as described above.

In this group $B$, the radiological tumour response, evaluated following the mRECIST target criteria at 3 months, was correlated with the perfused tumour dose and the perfused fraction of the total tumour volume, determined using ${ }^{90} \mathrm{Y}$ PET/CT and Simplicit ${ }^{90} \mathrm{Y}{ }^{\circledR}$.

\section{e. Statistical analysis}

Statistical analysis was performed using Stata/IC version 15.1. Descriptive statistics are reported as means and standard deviations for normally-distributed continuous variables or medians and ranges for asymmetrical distributions, and percentages for categorical variables. Normality was assessed with graphical representations (histogram, box plot). Homogeneity of variances was verified with Levene's test. Means were compared between the two groups with Student's t-test and asymmetrical distributions were compared with Mann-Whitney-Wilcoxon test. Frequencies were compared between the two groups with Fisher's exact test or Pearson's Chi-squared test, depending on the expected numbers. Number of successful attempts of treatment were compared with a generalized estimating equation (as some attempts were performed on the same patient). PFS and OS were analyzed using the Kaplan Meier method and compared with the logrank test.

\section{Results}

\section{a. Demographics and baseline characteristics}

Between February 2016 and December 2020, 66 patients underwent hepatic angiography and ${ }^{99} \mathrm{mTc}$ MAA scintigraphy in order to evaluate SIRT feasibility. Twenty-nine patients underwent work-up with standard dosimetry (group A) and 37 using personalized dosimetry (group B). Of the 32 evaluations in group A, 9 work-ups were unfavorable and the patients received treatment according to standard guidelines. Six patients had poor tumor targeting and 3 presented digestive extrahepatic uptake. Twentytwo patients received treatment $(75.86 \%)$, with one patient undergoing two treatments. 
In group B, a total of 47 work-ups were performed, with several patients undergoing more than one evaluation. Thirty failures were registered, mainly due to poor tumor targeting. Only 16 patients received treatment (43.24\%), with also one patient undergoing two radioembolizations on the same tumor.

The mains reasons for work-up failure are noted in the Flowchart (Figure 1).

Baseline characteristics of patients in groups A and B receiving SIRT are listed in Table 1. Demographics, tumor burden and liver function were similar for the two groups.

Table 1. Baseline characteristics 


\begin{tabular}{|c|c|c|c|}
\hline & Group A & Group B & p-value \\
\hline Baseline characteristics & $\mathrm{N}=22$ & $\mathrm{~N}=16 \mathrm{pts}$ & \\
\hline Age (years old) (Mean \pm SD ) & $72.00 \pm 9.07$ & $70.25 \pm 7.28$ & 0.53 \\
\hline Number patients that received treatment $(n, \%)$ & $\begin{array}{l}22 / 29 \\
(75.86 \%)\end{array}$ & $16 / 37(43.24 \%)$ & 0.008 \\
\hline Gender (male) (n, \%) & $\begin{array}{l}16 / 22 \\
(72.73 \%)\end{array}$ & $14 / 16(87.50 \%)$ & 0.43 \\
\hline Number of successful attempts of treatment $(n, \%)$ & $\begin{array}{l}23 / 32 \\
(71.88 \%)\end{array}$ & $17 / 39(43.59 \%)$ & 0.02 \\
\hline Cirrhosis (n, \%) & $\begin{array}{l}20 / 22 \\
(90.91 \%)\end{array}$ & $12 / 16(75 \%)$ & 0.22 \\
\hline Cirrhosis type (n, \%) & & & 0.77 \\
\hline ..Alcohol & $10 / 20(50 \%)$ & $6 / 12(50 \%)$ & 1.00 \\
\hline.. $\mathrm{HBV}$ & $1 / 20(5 \%)$ & $1 / 12(8.33 \%)$ & 1.00 \\
\hline.. $\mathrm{HCV}$ & $3 / 20(15 \%)$ & $3 / 12(25 \%)$ & 0.65 \\
\hline ..Other (combining factors) & $6 / 20(30 \%)$ & $2 / 12(16.67 \%)$ & 0.68 \\
\hline Child-Pugh (n, \%) & & & 1.00 \\
\hline..$A$ & $\begin{array}{l}16 / 18 \\
(88.89 \%)\end{array}$ & $11 / 12(91.67 \%)$ & \\
\hline.. $\mathrm{B}$ & $2 / 18(11.11 \%)$ & 1/12 (8.33\%) & \\
\hline ECOG Score $(n, \%)$ & & & 1.00 \\
\hline$\ldots 0$ & $\begin{array}{l}17 / 22 \\
(77.27 \%)\end{array}$ & $12 / 16(75 \%)$ & \\
\hline$\ldots 1$ & $5 / 22(22.73 \%)$ & $4 / 16(25 \%)$ & \\
\hline Previous treatment (n, \%) & & & 0.049 \\
\hline No previous treatment & $11 / 22(50 \%)$ & $13 / 16(81.25 \%)$ & \\
\hline At least one previous treatment & $11 / 22(50 \%)$ & $3 / 16(18.75 \%)$ & \\
\hline Previous treatment type (n, \%) & & & 0.47 \\
\hline ...SIRT/TACE & $5 / 11(45.45 \%)$ & $2 / 3(66.67 \%)$ & \\
\hline ....Systemic & $1 / 11(9.09 \%)$ & 1/3 (33.33\%) & \\
\hline ...Surgery & $1 / 11(9.09 \%)$ & $0 / 3(0 \%)$ & \\
\hline ...Multiples combination treatments & 4/11 (36.33\%) & $0 / 3(0 \%)$ & \\
\hline
\end{tabular}




\section{HCC-tumor load ( $n, \%)$}

\begin{tabular}{|c|c|c|c|}
\hline Infiltrative & 2/22 (9.09\%) & $2 / 16(12.50 \%)$ & 1.00 \\
\hline Satellite & $9 / 22(40.91 \%)$ & $5 / 16(31.25 \%)$ & 0.54 \\
\hline Ascites & $1 / 22(4.55 \%)$ & $1 / 16(6.25 \%)$ & 1.00 \\
\hline Portal vein invasion & $6 / 22(27.27 \%)$ & $4 / 16(25.00 \%)$ & 1.00 \\
\hline Portal hypertension & $\begin{array}{l}11 / 22 \\
(50.00 \%)\end{array}$ & $4 / 16(25.00 \%)$ & 0.12 \\
\hline Bile duct dilation & $4 / 22(18.18 \%)$ & $4 / 16(25.00 \%)$ & 0.70 \\
\hline Extrahepatic spread & $0 / 22(0 \%)$ & $2 / 16(12.50 \%)$ & 0.17 \\
\hline Number of nodules (Median [min-max] HCC) & $1[1-10]$ & $1[1-3]$ & 0.054 \\
\hline $\begin{array}{l}\text { Diameter of biggest nodule (mm) (Median [min- } \\
\text { max]) }\end{array}$ & 52 [17-163] & 65 [13-160] & 0.71 \\
\hline AFP Score $(n, \%)$ & & & 0.74 \\
\hline$<100$ & $\begin{array}{l}14 / 22 \\
(63.64 \%)\end{array}$ & $11 / 16(68.75 \%)$ & \\
\hline$\geq 100$ & $8 / 22(36.36 \%)$ & $5 / 16(31.25 \%)$ & \\
\hline $\operatorname{BCLC}(n, \%)$ & & & 1.00 \\
\hline.. $\mathrm{A}$ & $2 / 22(9.09 \%)$ & $2 / 16(12.50 \%)$ & \\
\hline. . B & $\begin{array}{l}14 / 22 \\
(53.64 \%)\end{array}$ & $10 / 16(62.50 \%)$ & \\
\hline..$C$ & $6 / 22(27.27 \%)$ & $4 / 16(25.00 \%)$ & \\
\hline Treatment characteristics & & & 0.26 \\
\hline ...Lobar & $\begin{array}{l}15 / 22 \\
(68.18 \%)\end{array}$ & $8 / 16$ (50.00\%) & \\
\hline ...Selective & $7 / 22(31.82 \%)$ & $8 / 16(50.00 \%)$ & \\
\hline
\end{tabular}

Three out of the 16 patients (18.75\%) in group B and 11 out of the 22 patients (50\%) patients in group $A$ had a previous treatment (not necessarily on the same lesion).

\section{b. Primary objectives}

No significant statistical difference was seen in terms of ORR between the two groups at 3 months evaluation. In group A objective response rate of treated nodules was of $68.42 \%$ (complete response [CR] of $10.53 \%$ and partial response [PR] of $57.89 \%$ ) and $87.5 \%$ (complete response $31.25 \%$ and partial 
response in $56.25 \%)$ in group $B(p=0.24)$. No response (stable disease [SD]) or progression (progressive disease [PD]) was observed in $31.58 \%$ in group $A$ and $12.5 \%$ in group $B$. Nevertheless, 1 patient that presented partial response was shown to have hepatic progression in a non-targeted area. The same situation was showed at 6 months evaluation (Table 2A).

Table 2. Overall response rate on target areas $(A)$ and best overall response (B - detailed response and $C$ - CR+PR and SD+PD) for the standard dosimetry group at 3 and 6 months, according to mRECIST.

CR- complete response, $\mathrm{PR}$ - partial response, SD- stable disease, $\mathrm{PD}$ - progressive disease according to mRECIST

DCR is defined as the composite of ORR and stable disease between patients in group A and B.

\begin{tabular}{|lllll|}
\hline \multicolumn{4}{|l|}{ A. mRECIST ORR for HCC treated nodules } \\
& 3 months & & 6 months \\
\hline Response $(\boldsymbol{n}, \%)$ & Group A & Group B & Group A & Group B \\
& N=19 & N=16 & N=10 & N=9 \\
\hline CR & $2 / 19(10.53 \%)$ & $5 / 16(31.25 \%)$ & $1 / 10(10 \%)$ & $3 / 9(33.33 \%)$ \\
\hline PR & $11 / 19(57.89 \%)$ & $9 / 16(56.25 \%)$ & $4 / 10(40 \%)$ & $5 / 9(55.56 \%)$ \\
\hline SD & $3 / 19(15.79 \%)$ & $1 / 16(6.25 \%)$ & $1 / 10(10 \%)$ & $0 / 9(0 \%)$ \\
\hline PD & $3 / 19(15.79 \%)$ & $1 / 16(6.25 \%)$ & $4 / 10(40 \%)$ & $1 / 9(11.11 \%)$ \\
\hline
\end{tabular}

\begin{tabular}{|c|c|c|c|}
\hline \multicolumn{4}{|c|}{ mRECIST ORR for HCC treated nodules } \\
\hline & Group A & Group B & p-value \\
\hline & $\mathrm{N}=19$ & $\mathrm{~N}=16$ & \\
\hline Response 3 months : n (\%) & & & 0.24 \\
\hline CR+PR & $13(68.4 \%)$ & $14(87.5 \%)$ & \\
\hline SD+PD & $6(31.6 \%)$ & $2(12.5 \%)$ & \\
\hline \multirow[t]{3}{*}{$D C R(C R+P R+S D) 3$ months: $n$ (\%) } & $16(84.2 \%)$ & $15(93.8 \%)$ & 0.61 \\
\hline & Group A & Group B & p-value \\
\hline & $\mathrm{N}=10$ & $N=9$ & \\
\hline Response 6 months : n (\%) & & & 0.14 \\
\hline CR+PR & $5(50.0 \%)$ & $8(88.9 \%)$ & \\
\hline SD+PD & $5(50.0 \%)$ & $1(11.1 \%)$ & \\
\hline
\end{tabular}




\begin{tabular}{|c|c|c|c|c|}
\hline \multicolumn{5}{|c|}{ A. BOR according to mRECIST overall } \\
\hline & 3 months & & 6 months & \\
\hline \multirow[t]{2}{*}{ Response (n, \%) } & Group A & Group B & Group A & Group B \\
\hline & $N=18$ & $\mathrm{~N}=15$ & $N=9$ & $N=9$ \\
\hline CR & 1/18 (5.56\%) & $5 / 15(33.33 \%)$ & $1 / 9(11.11 \%)$ & $3 / 9(33.33 \%)$ \\
\hline PR & $5 / 18(27.78 \%)$ & $7 / 15$ (46.67\%) & $1 / 9(11.11 \%)$ & $4 / 9(44.44 \%)$ \\
\hline SD & $5 / 18(27.78 \%)$ & $1 / 15$ (6.67\%) & $1 / 9(11.11 \%)$ & $0 / 9(0 \%)$ \\
\hline PD & 7/18 (38.89\%) & 2/15 (13.33\%) & $6 / 9(66.67 \%)$ & $2 / 9(22.22 \%)$ \\
\hline
\end{tabular}

\begin{tabular}{|c|c|c|c|}
\hline \multicolumn{4}{|c|}{ BOR according to mRECIST / overall } \\
\hline & Group A & Group B & p-value \\
\hline & $\mathrm{N}=18$ & $\mathrm{~N}=15$ & \\
\hline Response 3 months : $n$ (\%) & & & 0.007 \\
\hline CR+PR & $6(33.3 \%)$ & $12(80.0 \%)$ & \\
\hline \multirow[t]{3}{*}{ SD+PD } & $12(66.7 \%)$ & $3(20.0 \%)$ & \\
\hline & Group A & Group B & p-value \\
\hline & $\mathrm{N}=9$ & $\mathrm{~N}=9$ & \\
\hline Response 6 months : n (\%) & & & 0.06 \\
\hline CR+PR & $2(22.2 \%)$ & $7(77.8 \%)$ & \\
\hline SD+PD & $7(77.8 \%)$ & $2(22.2 \%)$ & \\
\hline
\end{tabular}

When we analyzed the response per patient, in terms of best overall response (BOR), a significant difference was showed with $80 \%$ versus $33.34 \%(p=0.007)$ for group $B$ and group $A$, respectively. This difference was equally observed at 6 months evaluation as showed in Table 2B. Nevertheless, this difference was not persistent for disease control rate at 3 months with $86.7 \%$ response in group $B$ versus $61.1 \%$ in group $A(p=0.15)$, nor at 6 months with $77.8 \%$ for group $B$ versus $33.3 \%$ for group $A$, despite a clear trend for a better efficacy of personalized treatment.

\section{c. Follow-up and survival}


The median time of follow-up was the same in the two groups, 21 months (range 3-55) in group A and 21 months (range 4-39) for the ones in group B.

The Kaplan-Meier curves of OS and PFS are depicted in Figure 2. Median OS in group A was 17.18 months (95\% Cl: 10.48 - not calculable) and 32.99 months (95\% Cl: 20.11 - not calculable) for group B. When compared, the two groups showed no statistical difference in terms of survival $(p=0.17)$. The same trend was noticed for PFS with a median time to progression in group A of 6.14 months ( $95 \% \mathrm{Cl}: 3.94$ $9.23)$ and 6.34 months (95\% Cl: $2.92-21.52)$ in group B.

\section{d. Safety and complications}

In the safety analysis, no mortality was registered at 30 days from treatment.

In group A, 6 out of 20 patients (30\%) evaluated at 1 months presented grade 1 or 2 clinical AEs (fatigue $33.33 \%$, abdominal pain $50 \%$ and nausea/vomiting $33.33 \%$ ). At three months evaluation, one patient also presented gastrointestinal bleeding due to newly appeared angiomas due to portal hypertension.

In group B, 7 patients out of $15(46.67 \%)$ that were evaluated at one month presented grade 1 or 2 clinical AEs (nausea $13.33 \%$, fatigue $13.33 \%$, abdominal pain $13.33 \%$, ascites $6.67 \%$, hemorrhagic duodenal ulcer $6.67 \%)$. The patient that received two treatments presented a hemorrhagic duodenal ulcer one month after his second treatment. At 3 and 6 months only 3 and 1 patients respectively, still presented clinical toxicities. The latter had grade 3 ascites that required repeated paracentesis.

The most common biochemical toxicity at 1 month in group A was hyperbilirubinemia (20\%). This persisted at 3 months, with one patient presenting Grade 3 toxicity.

However, the most common biochemical toxicity for group B was decrease albumin at 1 month evaluation (45.45\%). No grade 3 or 4 toxicities were shown. No clinical adverse events (AEs) were reported at day 1 after treatment.

In terms of radiological AEs, 5 peritumoral ischemic lesions were shown on CE imaging at three months in group $B$, with no repercussion on the hepatic function. No biliary injury was noticed. In the patients of group A, 6 ischemic injuries were observed. One patient presented biliary dilation with elevation of blood cholestasis.

\section{e. Dose-response link evaluation in groups $A$ and $B$, and retrospective recalculation of activity to be injected in group A using Simplicit ${ }^{90} \mathrm{Y}^{\circledR}$}

Regarding the group A, in order to compare the administered activity according to standard dosimetry and the activity that would have been recommended by the Simplicit ${ }^{90} \mathrm{Y} \circledast$ software, 18 patients were

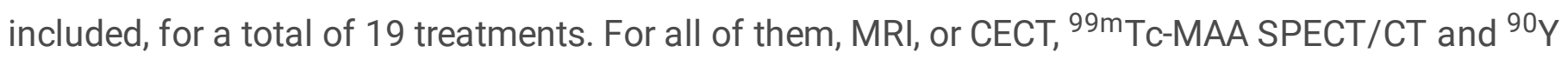


BECT/CT were available. Among these 19 treatments evaluated by mRECIST (target) criteria at 3 months, 11 treatments induced a PR and 2 a CR. In these responders, the ${ }^{90} \mathrm{Y}$ BECT/CT-based dosimetry showed 7 patients receiving less than $205 \mathrm{~Gy}$ and 6 patients receiving more than $205 \mathrm{~Gy}$ in the perfused tumour (188 Gy on average) (Figure 3). For these latter 6 patients, the comparison between the administered activity and the recommended activity estimated retrospectively using Simplicit ${ }^{90} Y{ }^{\circledR}$ showed that 5 patients received an activity that was higher than recommended (79\% mean difference) (Figure 4), but, with a dose to the non-tumoral liver staying below 70 Gy except for 1 patient (44 Gy on average) ${ }^{20}$.

Among the 7 responding patients receiving less than 205 Gy in the perfused tumor, 2 patients showed visible areas of necrosis before treatment on the anatomical images used.

Among the 6 treatments inducing no response, 3 patients progressed and 3 patients were stable. All of these patients received less than $205 \mathrm{~Gy}$ to the perfused tumor, based on a ${ }^{90} \mathrm{Y} \mathrm{BECT/CT-based} \mathrm{dosimetry,}$ except one (Figure 3). In the latter patient, microspheres were concentrated non-homogenously with a well-defined hot spot and undertreated parts (Figure 5). The comparison between the administered activity and the recommended activity calculated using Simplicit ${ }^{90} Y{ }^{\circledR}$ showed that the other 2 patients who progressed received less activity than recommended (51\% mean difference). The results are the same for 2 out of 3 patients who were stable ( $47 \%$ mean difference for these 2 out of 3 ) (Figure 4 ).

Ten patients of the group B underwent post treatment ${ }^{90} \mathrm{Y}$ PET/CT, thus allowing dose-response analysis to be conducted. At the 3 months imaging evaluation 8 treatments induced a response (5PR and $3 \mathrm{CR}$ ). Among the 2 treatments inducing no response, 1 patient progressed and 1 patient was stable (Figure 6 ).

In the group A (with BECT/CT-based dosimetry), a threshold of $175 \mathrm{~Gy}$ appears to be the cut-off value to reach response, at least partially (Figure 3), except for 1 patient who progressed with a well, but nonhomogenously perfused tumor (Figure 5). In the group B (with PET-CT based dosimetry), the perfused fraction of the total tumor volume seems to play a role too, because we encountered a patient in progression after 3 months (regarding the targeted tumor following mRECIST criteria) in whom the covering fraction was $80 \%$. Reaching more than $90 \%$ for the covering fraction and 400 Gy for the entire tumor dose ensure response with an important proportion of total responses in this group (60\%) (Figure $6)$.

\section{Discussion}

This retrospective trial compared the objective response rate for treated nodules as well as the best overall response in our population of HCC patients that underwent treatment with radioembolization using standard predictive dosimetry versus personalized predictive dosimetry. For patients treated after standard-dosimetry-based simulation, the activity that would have been administered by SIRT determined using a personalized dosimetry software with multicompartment MIRD technique (Simplicit ${ }^{90} \mathrm{P}$ ) was compared to the activity actually administered determined by the classical non-compartmental dosimetry planning, using a target volume based on the ${ }^{99 \mathrm{~m}}$ TC-MAA SPECT (patients treated before the acquisition 
of Simplicit $\left.{ }^{90} Y{ }^{\circledR}\right)$. To the best of our knowledge, this is the first study that addressed the potential changes due to personalized predictive dosimetry in treatment administration in a retrospective cohort.

In our study, there was no significant statistical difference when comparing the two groups in terms of response rate per treated nodule (ORR), despite a trend in favor of personalized dosimetry ( $87.5 \%$ for personalized dosimetry versus $68.4 \%$ for standard dosimetry at 3 months, $p=0.24$ ). These discordant results are probably due to the small size of our two groups population. By contrast, in the per patient analysis, there was a clear benefit of personalized dosimetry in terms of BOR at 3 months ( $80 \%$ versus $33.3 \%, p=0.007)$ and 6 months post-treatment $(77.8 \%$ versus $22.2 \%, p=0.06)$.

The response rate we report is slightly higher than the results published recently in the randomized, multicenter DOSISPHERE-01 study ${ }^{23}$ using glass microspheres. In this study by Garin et al, a significant statistical difference was shown between objective response rates in favor of personalized predictive dosimetry compared to standard predictive dosimetry $(71 \%$ versus $36 \%)$. However, we note that the diameter of tumors in our study was smaller, with a median of $65 \mathrm{~mm}$, whilst in the DOSISPHERE-01, at least one nodule had minimum $70 \mathrm{~mm}$. Large tumor size is well known to have a strong negative impact on response and overall survival after locoregional therapies ${ }^{24}$. This might partially explain the better results in our cohort.

Regarding toxicity, we observed a good safety profile in both groups with only one grade 3 biological toxicity. More importantly, due to the excellent tolerance and toxicity profile, almost all patients in our study were able to have sequential locoregional or systemic treatment. The preservation of the liverfunction is more than essential ever since the development of multiple systemic treatments that improve the overall survival of HCC patients. The final results of the IMBRAVE 150 trial comparing the efficacy of the atezolizumab plus bevacizumab combination versus sorafenib for unresectable HCC showed a median overall survival (OS) of 19.2 vs. 13.4 months; stratified HR $0.66,95 \% \mathrm{Cl}[0.52,0.85] ; \mathrm{p}=0.0009$ ) with excellent tolerance ${ }^{25}$. Moreover, $48 \%$ of patients receiving the combination treatment and $52 \%$ of those in the Sorafenib arm had already underwent a local therapy before enrollment in the study, suggesting that a locoregional treatment does not prevent sequential treatment.

The DOSISPHERE-01 trial was a breakthrough for SIRT after 2 negative phase III trials (SARAH and SIRveNIB) and one phase II trial (SORAMIC) comparing or evaluating the addition of SIRT to Sorafenib. In terms of overall survival, personalized predictive dosimetry showed an important added value, with 26.6 months for the intention-to-treat population compared to 9.9 months in the SARAH trial and 11.3 months in the SIRveNIB trial. In the SORAMIC trial, the SIRT + sorafenib arm showed a median OS of 12.1 months, with no statistical difference from the Sorafenib arm of 11.4 months. Our study has shown an improvement of OS with personalized dosimetry, 33 months versus 17.2 months for patients treated after standard-dosimetry-based simulation. However, despite the advantage of personalized predictive dosimetry in terms of response, the survival was not impacted. This came as a surprise as a recent study evaluating the relationship between tumor absorbed dose, survival and tumor response in locally advanced inoperable HCC based on the SARAH study population showed that patients who received a 
higher dose (a threshold of $100 \mathrm{~Gy}$ for resin microshperes) had longer survival, 14.1 months [95\% Cl: 9.6 months, 18.6 months] vs 6.1 months [ $95 \% \mathrm{Cl}: 4.9$ months, 6.8 months], respectively; $p=0.001)^{26}$. However, this might be also explained by our small population sample included in our study.

With the use of personalized predictive dosimetry, we obtain a better selection of patient that could benefit from SIRT, avoiding inefficient radiotherapies and thus unnecessary dose deposition, an obvious critical point in patient radioprotection. However, this leads to an increase of work-up failure. A higher number of patients were not suitable for SIRT after simulation with the use of Simplicit90Y®, $24.1 \%$ for group A versus $56.8 \%$ for group B. This point is often overlooked and yet, it is important to point out since patients might undergo an unnecessary hepatic angiography that represents an invasive procedure inducing stress. This drop-out in our study is illustrated in Table 1. In the standard predictive dosimetry group $75,86 \%$ of patients received treatment, compared to $43,24 \%$ in the personalized predictive dosimetry group, despite more than one work-up attempts. The principal explanation was shown to be poor tumor targeting in $50 \%$ of cases, resulting from the more precise multi-compartmental dosimetry approach available with Simplicit ${ }^{90} \mathrm{Y}$. As mentioned above, this can be interpreted as an advantage since it avoids a potentially toxic treatment in patients that have already an abnormal liver function. As a matter of fact, the toxicity profile ends up particularly favorable in our study. Of notice, high work-up failure is not observed when performing transarterial chemoembolization (TACE), particularly with the latest developments in drug eluting beads (DEB-TACE) and new catheters (e.g., balloon microcatheter). A recent prospective feasibility study of balloon-occluded chemoembolization using polyethylene glycol DEBs loaded with Doxorubicin, performed at our center, was recently published and has showed a $100 \%$ rate of technical success ${ }^{27}$. With adequate selection, median OS after TACE may reach 48 months as shown in a large retrospective study ${ }^{28}$.

In group A, the comparison between the administered activity and the recommended activity calculated using a personalized dosimetry software with multicompartmental (MIRD) approach showed that the patients who progressed received less activity than recommended (Figure 4) or that this activity was not adequately distributed (Figure 5). Our results indicate that reaching a minimal absorbed dose criteria increases the efficacy of SIRT. They support the use of a more personalized predictive dosimetry instead of the classical non-compartmental MIRD dosimetry for treatment planning. This is in line with the notion that a minimal absorbed dose of a minimum $205 \mathrm{~Gy}$ to the lesion is required to achieve an optimal response for SIRT with glass microspheres ${ }^{19,20}$.

Note that taking into account areas of necrosis in the tumor absorbed dose calculation does not provide an estimate of the dose to the viable tumor, as mentioned by Garin et al.

Dose-response link evaluations, in both groups, shows clearly that a total response cannot be reached if the perfused fraction of the total tumor volume is not enough and about $100 \%$. Giving enough dose to a sufficient tumor volume fraction ensures at least a partial response. 
Our study has several limitations, the most important being the small number of patients and its retrospective nature. Furthermore, a bias is to be taken into account due to the fact that some patients had already undergone previous treatments on the treated tumors. Moreover, the groups have been treated in two successive periods. Therefore, the benefit of the experience acquired may have influenced our results for the patients in group B. This effect is mitigated by the fact that the most sensitive part of the protocol that is subjected to a learning curve, that is the angiographic procedure has been performed by experienced radiologists (between 5-10 years' experience of hepatic angiography practice).

\section{Conclusion}

Our data contributed to display that multi-compartmental dosimetry, more personalized dosimetry, plays a key role to estimate in advance the efficacy of SIRT during simulations. A higher BOR can be reached by (more) personalized dosimetry over standard one and this difference may explain disappointing data previously reported, indicating that a (more) personalized dosimetry is needed for SIRT planning in the treatment of HCC.

This study also contributed to confirm the need to go further. Multi-compartmental dosimetry is a first and important step but personalizing dosimetry leads to voxel-wise dosimetry allowing intra-tumoral dose deposition heterogeneity estimation. Guidelines need larger studies to evolve following this way.

\section{Declarations}

\section{Author's contributions:}

A-M Bucalau and B. Collette: investigation and writing original draft; I. Tancredi, M Vouche and N. Trotta: procedures; M. Pezzullo: imaging validation; J. Bouziotis: statistical analysis; R. Moreno-Reyes: evaluation of dosimetry and methodology; Jean Luc Van Laethem: reviewing; G. Verset: conceptualization and supervision.

\section{Institutional review board statement:}

Hôpital Erasme Ethics Committee P2021/221

\section{Conflict of interest:}

regarding this study the conflict of interest are:

A-M Bucalau: no conflicts to declare; B. Collette: no conflicts to declare. I. Tancredi : consultant for Terumo Europe NV and BTG International; M. Vouche : ; M. Pezzullo: no conflicts to declare; J. Bouziotis: no conflicts to declare; R. Moreno-Reyes: no conflicts to declare; N. Trotta; no conflicts to declare; JL. Van Laethem: no conflicts to declare; G. Verset: consultant for Terumo Europe NV and BTG International.

\section{References}


1. Galle, P. R. et al. EASL Clinical Practice Guidelines: Management of hepatocellular carcinoma. J. Hepatol. (2018) doi:10.1016/j.jhep.2018.03.019.

2. Heimbach, J. K. et al. AASLD guidelines for the treatment of hepatocellular carcinoma. Hepatology (2018) doi:10.1002/hep.29086.

3. Forner, A., Reig, M. \& Bruix, J. Hepatocellular carcinoma. The Lancet (2018) doi:10.1016/S01406736(18)30010-2.

4. Sangro, B. \& Salem, R. Transarterial chemoembolization and radioembolization. Semin. Liver Dis. (2014) doi:10.1055/s-0034-1394142.

5. Salem, R. et al. Radioembolization for Hepatocellular Carcinoma Using Yttrium-90 Microspheres: A Comprehensive Report of Long-term Outcomes. Gastroenterology (2010) doi:10.1053/j.gastro.2009.09.006.

6. Salem, R., Mazzaferro, V. \& Sangro, B. Yttrium 90 radioembolization for the treatment of hepatocellular carcinoma: Biological lessons, current challenges, and clinical perspectives. Hepatology (2013) doi:10.1002/hep.26382.

7. Hilgard, P. et al. Radioembolization with yttrium-90 glass microspheres in hepatocellular carcinoma: European experience on safety and long-term survival. Hepatology (2010) doi:10.1002/hep.23944.

8. Sangro, B. et al. Survival after Yttrium-90 resin microsphere radioembolization of hepatocellular carcinoma across Barcelona clinic liver cancer stages: A European evaluation. Hepatology (2011) doi:10.1002/hep.24451.

9. Smits, M. L. J. et al. Holmium-166 radioembolisation in patients with unresectable, chemorefractory liver metastases (HEPAR trial): A phase 1, dose-escalation study. Lancet Oncol. (2012) doi:10.1016/S1470-2045(12)70334-0.

10. van Roekel, C. et al. Evaluation of the Safety and Feasibility of Same-Day Holmium-166 Radioembolization Simulation and Treatment of Hepatic Metastases. J. Vasc. Interv. Radiol. (2020) doi:10.1016/j.jvir.2020.01.032.

11. Vilgrain, V. et al. Efficacy and safety of selective internal radiotherapy with yttrium-90 resin microspheres compared with sorafenib in locally advanced and inoperable hepatocellular carcinoma (SARAH): an open-label randomised controlled phase 3 trial. Lancet Oncol. (2017) doi:10.1016/S1470-2045(17)30683-6.

12. Chow, P. K. H. et al. SIRveNIB: Selective internal radiation therapy versus sorafenib in Asia-Pacific patients with hepatocellular carcinoma. J. Clin. Oncol. (2018) doi:10.1200/JC0.2017.76.0892.

13. Ricke, J. et al. The impact of combining Selective Internal Radiation Therapy (SIRT) with sorafenib on overall survival in patients with advanced hepatocellular carcinoma: The SORAMIC trial palliative cohort. Ann. Oncol. (2018) doi:10.1093/annonc/mdy149.028.

14. Dezarn, W. A. et al. Recommendations of the American Association of Physicists in Medicine on dosimetry, imaging, and quality assurance procedures for $90 \mathrm{Y}$ microsphere brachytherapy in the treatment of hepatic malignancies. Med. Phys. (2011) doi:10.1118/1.3608909. 
15. Grosser, O. S. et al. Intrahepatic activity distribution in radioembolization with yttrium-90-labeled resin microspheres using the body surface area method - A less than perfect model. J. Vasc. Interv. Radiol. (2015) doi:10.1016/j.jvir.2015.07.021.

16. Ho, S. et al. Tumour-to-normal uptake ratio of $90 \mathrm{Y}$ microspheres in hepatic cancer assessed with 99TCm macroaggregated albumin. Br. J. Radiol. (1997) doi:10.1259/bjr.70.836.9486047.

17. Ho, S. et al. Clinical evaluation of the partition model for estimating radiation doses from yttrium-90 microspheres in the treatment of hepatic cancer. Eur. J. Nucl. Med. (1997) doi:10.1007/s002590050055.

18. Garin, E., Rolland, Y., Laffont, S. \& Edeline, J. Clinical impact of 99mTc-MAA SPECT/CT-based dosimetry in the radioembolization of liver malignancies with $90 \mathrm{Y}$-loaded microspheres. Eur. J. Nucl. Med. Mol. Imaging (2016) doi:10.1007/s00259-015-3157-8.

19. Garin, E. et al. High impact of macroaggregated albumin-based tumour dose on response and overall survival in hepatocellular carcinoma patients treated with 90Y-loaded glass microsphere radioembolization. Liver Int. (2017) doi:10.1111/liv.13220.

20. Gnesin, S. et al. Partition model-based 99mTc-MAA SPECT/CT predictive dosimetry compared with 90Y TOF PET/CT posttreatment dosimetry in radioembolization of hepatocellular carcinoma: A quantitative agreement comparison. J. Nucl. Med. (2016) doi:10.2967/jnumed.116.173104.

21. Lencioni, R. \& Llovet, J. M. Modified recist (mRECIST) assessment for hepatocellular carcinoma. Seminars in Liver Disease (2010) doi:10.1055/s-0030-1247132.

22. U.S. DEPARTMENT OF HEALTH AND HUMAN SERVICES. Common Terminology Criteria for Adverse Events (CTCAE).v.5.0. Cancer Therapy Evaluation Program (CTEP) (2017).

23. Garin, E. et al. Personalised versus standard dosimetry approach of selective internal radiation therapy in patients with locally advanced hepatocellular carcinoma (DOSISPHERE-01): a randomised, multicentre, open-label phase 2 trial. Lancet Gastroenterol. Hepatol. (2021) doi:10.1016/S2468-1253(20)30290-9.

24. Kadalayil, L. et al. A simple prognostic scoring system for patients receiving transarterial embolisation for hepatocellular cancer. Ann. Oncol. (2013) doi:10.1093/annonc/mdt247.

25. Finn, R. S. et al. Atezolizumab plus Bevacizumab in Unresectable Hepatocellular Carcinoma. N. Engl. J. Med. (2020) doi:10.1056/nejmoa1915745.

26. Hermann, A. L. et al. Relationship of tumor radiation-absorbed dose to survival and response in hepatocellular carcinoma treated with transarterial radioembolization with 90Y in the SARAH study. Radiology (2020) doi:10.1148/radiol.2020191606.

27. Bucalau, A.-M. et al. Balloon-occluded chemoembolization for hepatocellular carcinoma: a prospective study of safety, feasibility and outcomes. Hepatic Oncol. (2021) doi:10.2217/hep-20200022.

28. Burrel, M. et al. Survival of patients with hepatocellular carcinoma treated by transarterial chemoembolisation (TACE) using Drug Eluting Beads. Implications for clinical practice and trial design. J. Hepatol. (2012) doi:10.1016/j.jhep.2012.01.008. 


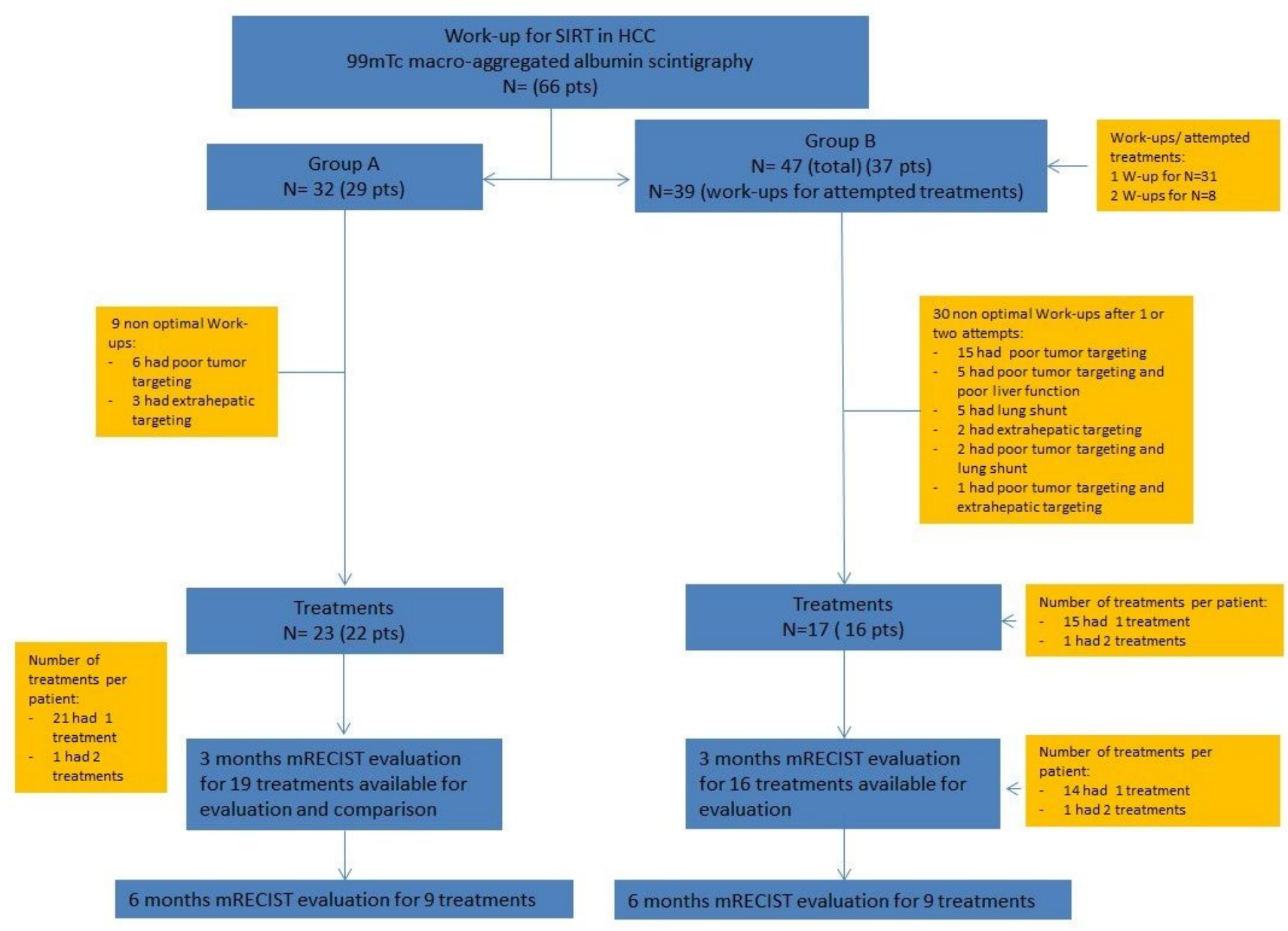

\section{Figure 1}

Flowchart of the study (Group A-patients that received SIRT after standard-based-dosimetry simulation; Group B- patients that received SIRT after personalized-based-dosimetry simulation). 
A

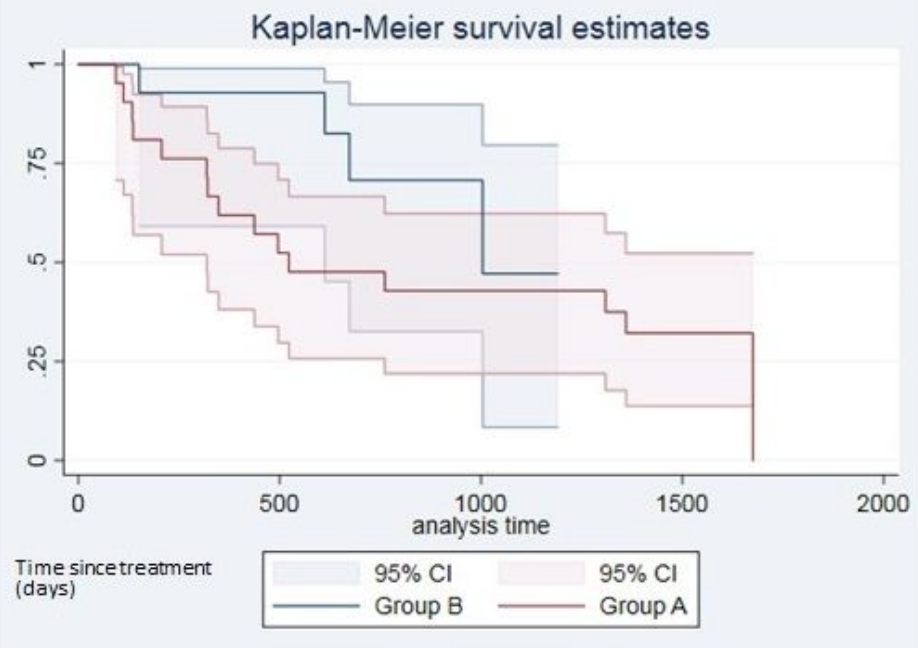

B

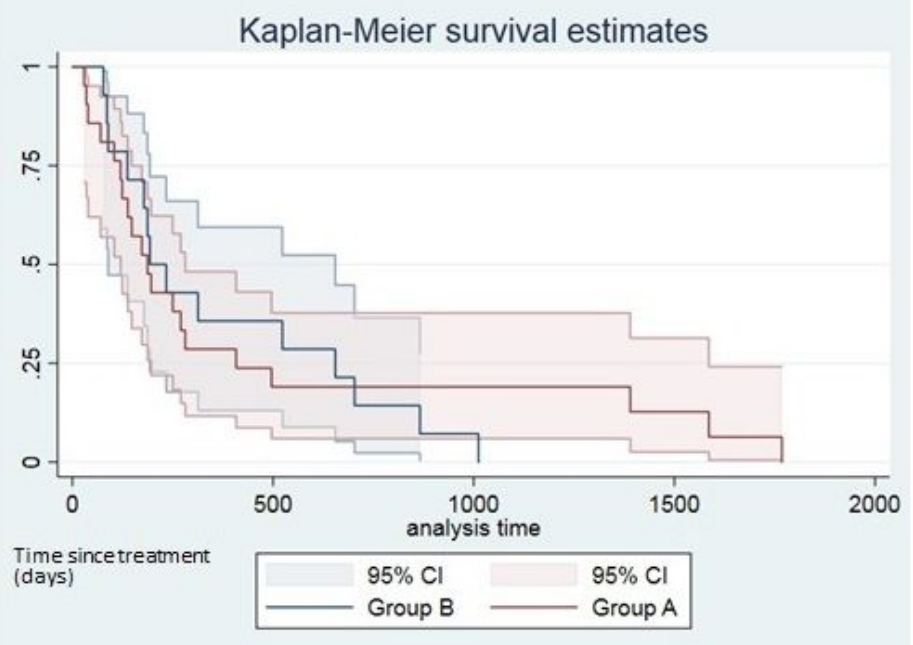

\section{Figure 2}

Kaplan-Meier curves for overall survival (OS) (A) and progression-free survival (PFS) (B). No statistically significant difference was seen between group $A$ and $B$ in OS or PFS. 


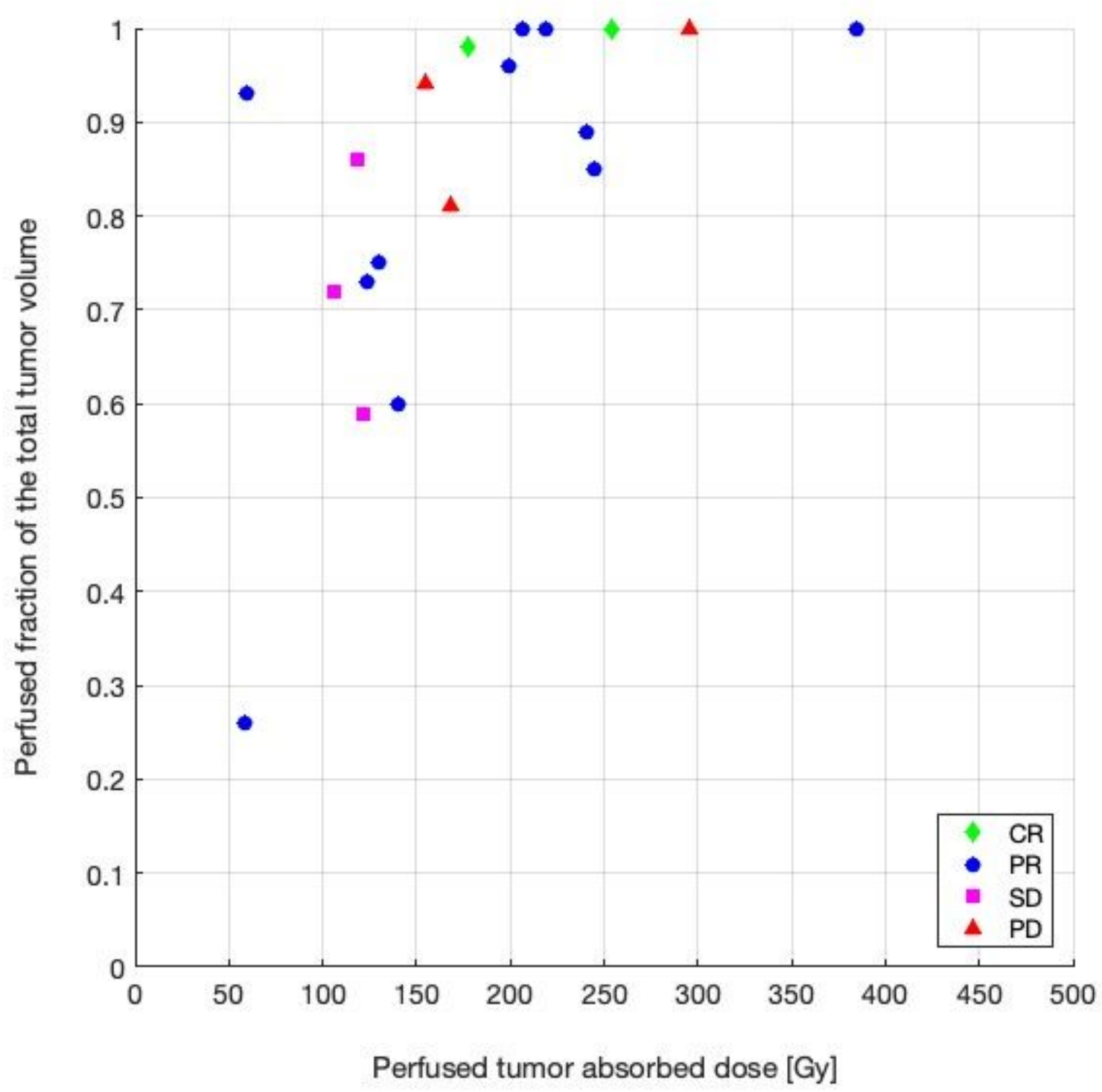

Figure 3

Dot plot of perfused fraction of the total tumor volume according to perfused tumor adsorbed dose and tumor response evaluated following mRECIST (target) criteria at 3 months, in patients from group A with BECT-CT-based dosimetry 


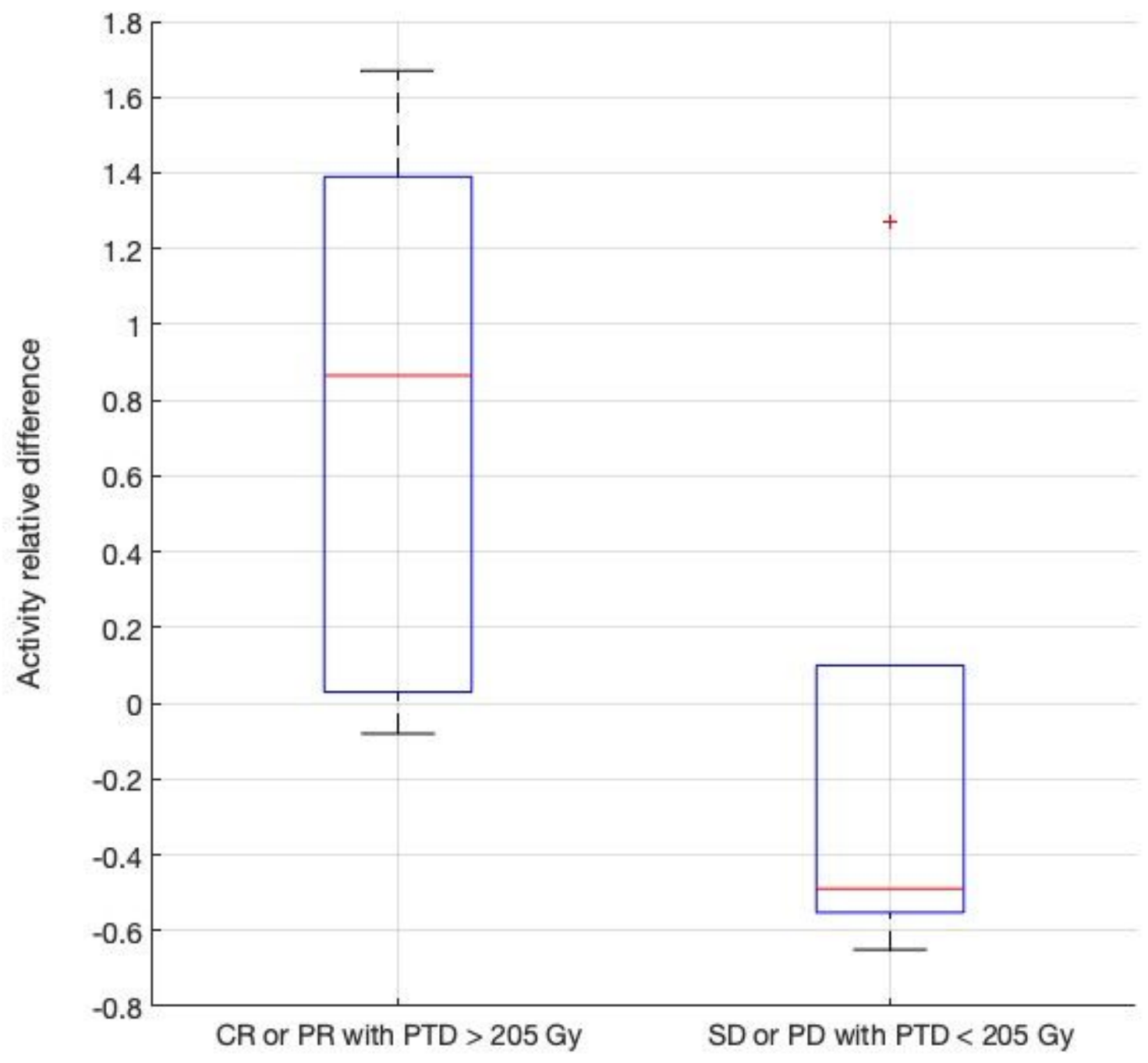

Figure 4

Box plot showing the distribution of activity relative differences (between activity to be administered following personalized dosimetry and activity actually administered following classical standard approach) in 2 subgroups of group A (complete or partial response evaluated at 3 months following mRECIST target criteria with perfused tumor dose above 205 Gy versus stable or progression disease with perfused tumor dose under $205 \mathrm{~Gy}$ ). 


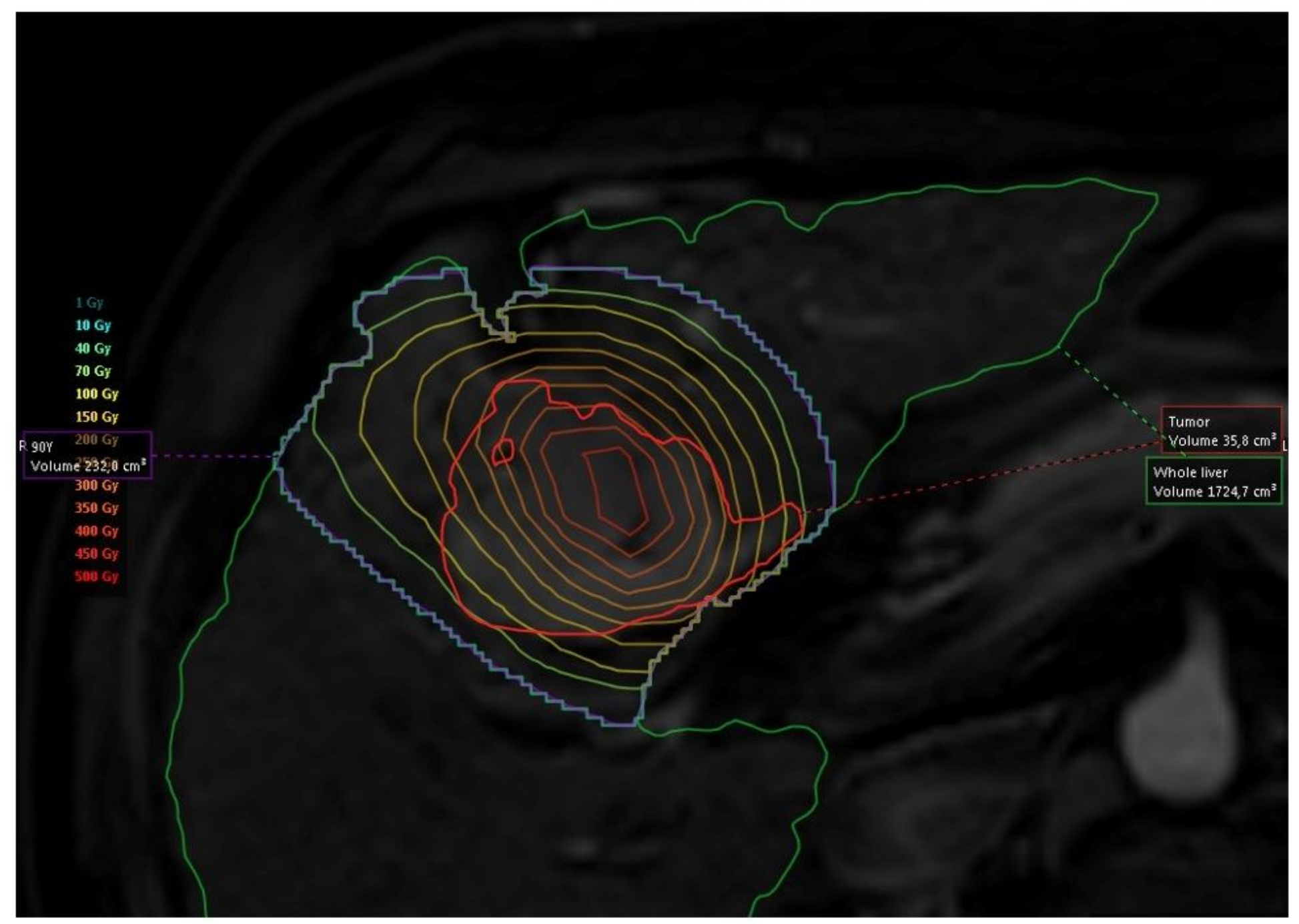

\section{Figure 5}

Isodose curves from 90Y BECT on MRI images (T2 sequence) in a 61-year-old male patient suffering from an HCC well perfused but non-homogeneously with undertreated parts (under $205 \mathrm{~Gy}$ ) 


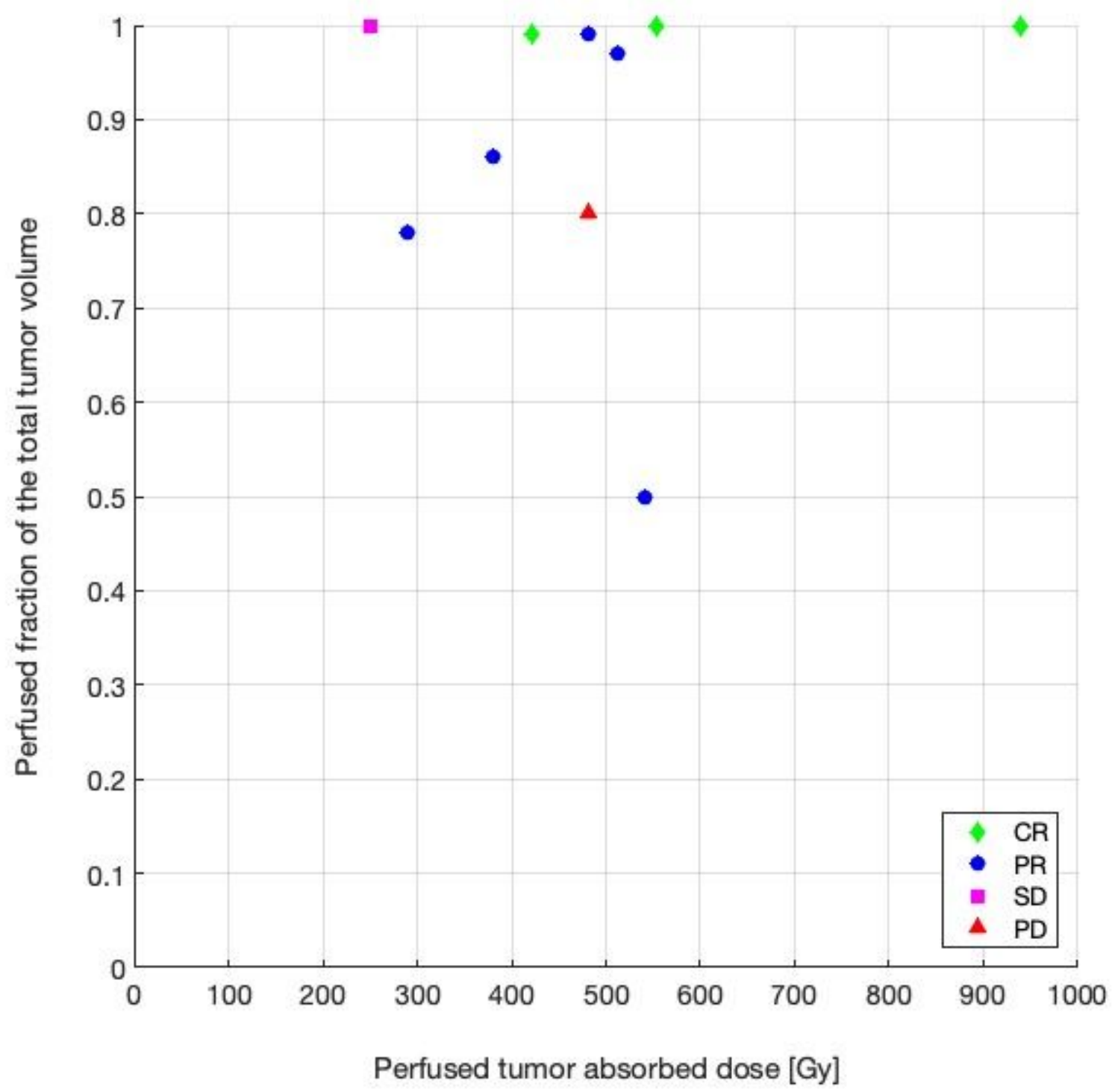

Figure 6

Dot plot of perfused fraction of the total tumor volume according to perfused tumor adsorbed dose and tumor response evaluated following mRECIST (target) criteria at 3 months, in patients from group B with PET-CT-based dosimetry 